\title{
Ce qu'on se dit le dimanche
}

\author{
Gail Scott, Nicole Brossard, Louise Cotnoir, Louky Bersianik, \\ France Théoret, Louise Dupré
}

\section{What We Talk About On Sundays}

Conversation and dialogue are each assigned a place in our Sunday meetings. And we six women writers takeas much pleasure in the intellectual mutual respect and rigour we apply to reading and discussing each other's work, i.e., the 'coffee' part of the meeting, as we do in the 'conversational' part later, accompanied by wine and good food. The following texts come out of our discussion on la post-modernité. For in Quebec the period of la modernité (generally translated in English by post-modernism or modernity) is said to have been eclipsed by la postmodernité. Since la modernité was so important for the women here, introducing concepts like fiction-theory, the reabsorbing of theory into the text, the baroque forms that signal the latter, are greeted with mixed feelings by women here.

Il me semble que la conversation et le dialogue ont chacun leur place dans notre groupe de six écrivaines. A nos réunions, qui ont souvent lieu le dimanche, c'est le dialoguequi passe en premier, accompagnéde bon caféfort. Plus tard dans la journée, nous nous appliquerons à la partie plaisir de la réunion, au vin, au bon manger, et bien sûr, à la conversation. Mais on tient à structurer la première partie de ces rencontres, à partir des textes écrits, afin de mieux nous concentrer sur les questions théoriques qui nous solicitent: la femme comme sujet, l'engagement féministe, la narration, pour en nommer quelques unes. Pour nous toutes, je crois, ce désir de travail rigoureux tient de notre respect réciproque de la pensée des autres membres du groupe. De plus, nous qui sommes écrivaines, travailleuses, mères dans certains cas, disposons de peu de temps pour se voir. Il faut en profiter au maximum! Souvent pendant la partie plaisir de la réunion, la conversation tourne encore autour de nos préoccupations théoriques, car nos échanges prennent fréquemment un 
élan difficile à freiner. Naturellement, chacune de nous a sa façon de dialoguer, de converser, et de mêler les deux... Je nous vois autour d'une table: Louise Cotnoir, son esprit vif, sensible, son engagement inébranlable, France Théoret, qui sait parler aussi bien qu'écouter avec une attention, une précision exemplaires, Louky Bersianik, critique infatigable de tout ce qui relève du domaine symbolique, Nicole Brossard, optimiste, intégrale, avec son flair impressionant pour l'essentielle; Louise Dupré, dont les phrases limpides dévoilent une grande intuition théorique ... et moi.

Les textes qui suivent sont des textes rédigés à partir d'une de nos discussions, cette fois sur le féminisme et la post-modernité. Au Québec, actuellement, la modernité, si importante pour l'écriture au féminin, semble, aux dires de certains, se faire sonner le glas. Cette modernité, qui a ouvert des brèches si importantes pour les femmes avec son insistence sur l'éclatement du genre, la déconstruction du sujet unaire, et le côté réfléxif de l'écriture, serait en train de céder sa place à une écriture post-moderne où la théorie serait réabsorbée, où la notion de progrès qui soustend la modernité serait dépassée par une ambiance (plus décadante?) de fin de siècle. Mais ce déplacement théorique offrirait aussi de nouvelles possibilités narratives, mettrait en relief aussi un certain tragique, précurseur peut-être de nouvelles visions. Qu'en pensons-nous, les femmes du groupe? Vous verrez que nous ne sommes pas toujours d'accord!

(Pour ne pas trop mêler les lectrices anglophones, la modernité est généralement traduite par 'postmodernism' en anglais. Quand à la postmodernité, je ne vois pas d'équivalent philosophique en anglais. Mais je dirais que la new-narrative anglo-américaine (Kathy Acker, Lynne Tillman, entre autres) semble imprégnée d'un esprit similaire à certaines expériences 'postmodernes' en français.) 


\section{Les pas juxtaposés du désir}

\section{Juxtaposed Steps of Desire}

La post-modernité has to do with contradictory feelings, nostalgia, disoriented desire, a kind of faceless tragedy despite the parade of bodies, of expressions. Paradoxically, this tragedy permits a certain hope and questioning. Partly, this is because the mythic sex-appeal of Man has lost its credibility. And la post-modernité is only viable inasmuch as the feminine is generated at the heart of this empty period.

La post-modernité, c'est affaire de sensations fortes, de sentiments contradictoires, de nostalgie; approche dérèglementée du désir, désir désorienté. Avènement d'un écart entre le savoir et l'émotion, la post-modernité ressemble à un théâtre dans lequel les subjectivités se croisent, vont et viennent, toutes plus performantes les unes que les autres, tandis que quelques souffleurs s'épuisent du fond de leur fosse, à murmurer le texte original, un texte dont la représentation semble désormais impossible. Il y a là un tragique sans visage malgré $l^{\prime}$ abondance de corps et d'expressions. Paradoxalement, ce tragique garantit du questionnement et de l'espoir.

La post-modernité, $c^{\prime}$ est l'amortissement $d^{\prime}$ 'un deuil non-consenti à l'histoire, c'est le pensé traumatique de l'histoire dissoute en son accélération: soudaine apesanteur. $C^{\prime}$ est l'aventure du sujet contraint sans avertissement à la légèreté, contraint surtout à une forme de connaissance à laquelle il répugne: l'intuition. Il y a là une posture féminine qui n'est pas sans gêner les intellectuels, qui avantage, comme c'est souvent le cas, les créateurs, qui semble relativement confortable pour les femmes. En fait, si l'intuition semble être le seul recours que nous ayons pour comprendre les mondes simultanément déserts et encombrés que nous habitons, $c^{\prime}$ est quela figure médiatique de l'Homme ainsi que le construit imaginaire qui en assurait le sexappeal mythique ne sont plus crédibles. De cette grande figure de prédateur, il ne reste qu'une bouche désabusée qui fait la moue.

Vu sous cet angle, la post-modernité est un 'grand down' masculin. Vu sous un autre angle, elle est un moment creux dans lequel se joue pourtant 1 'avenir des méthodes cognitives, des valeurs, des mythes et des métaphores qui détermineront de nouveaux rapports à la nature, 
à l'origyne et à la mort. La post-modernité n'est viable qu'en ce sens, viable que s'il y a du féminin qui génère de l'imaginaire au coeur du temps long que constitue ce creux tridimentionnel.

Nicole Brossard 


\section{La post-modernité? un leurre}

\section{La Post-modernité: A Trap}

The urge to get rid of rebellious modernité so quickly wouldn't have anything to do with the fact that it was the writing space in which women were able to write their dreams, their bodies, their hopes? La post-modernité is a sidetracking of women's innovations in writing, a sidetracking that underlines male complicity with a certain anti-life way of thinking....

Avec la post-modernité j'ai l'impression qu'on a voulu 'jeter le bébé' de la modernité avec l'eau du bain ... Qu'on a voulu sortir trop tôt de cette modernité si explosive, qu' on a voulu la courcircuiter ... Pourquoi? Parce que les femmes ont pu y formuler leurs rêves? Parce que les femmes y ont écrit autrement leur corps, leurs espoirs, leur imaginaire? La post-modernité vient désamorcer leur travail de sape, leurs revendications, leurs façons de 'réinventer la vie'.

Que dit la post-modernité en écriture au Québec? Elle fait un 'Colloque sur la mort du genre' (cf. La Nouvelle Barre du Jour)!!! De quel genre est-il question, sinon du genre MASCULIN?!? Personne dans le contexte baroque actuel, où les priorités vont à l'économie, à la natalité et aux armes nucléaires, $n^{\prime}$ ose dire, derrière le voile diaphane des médias, que ce sont là des questionnements suscités, il y a longtemps, par les féministes ... Que dans leurs écritures, les auteures se sont attaqué à tous les pouvoirs qui sont responsables $\mathrm{du}$ 'crash' économique, social et politique dont les thèmes de 'désenchantement, de désastre, de mort' font les beaux textes de cette post-modernité ... MALE ... LA LEUR! La post-modernité est un détournement de fonds des innovations des femmes en écriture (le mélange des genres, les néologismes, l'écriture fragmentaire etc.). La post-modiernité exprime la complaisance mâle pour le mortifère. La post-modernité est l'ultime expression de sa pulsion suicidaire.

Comment les auteures féministes peuvent-elles encore se laisser avoir par un tel leurre?! Je garde la posture de la modernité parce qu'elle m'offre la possibilité de transformer l'écriture et la société, de les féminiser. 


\section{Note pour un 'Alphabet des revelations' *}

\section{Notes for an 'Alphabet of Revelations'}

The categories of modernité and post-modernité are defined by male intellectuals and I' $m$ not sure women should accept these categories. Among other things it is looking to reconstitute (chercheles ensembles) what has been fragmented, in conflict. La post-modernité signals a swinging of the pendulum, back, I bet, towards a patriarchal grid.

Il serait commode de considérer la post-modernité comme un film projeté à l'envers, où les failles volontaires de la modernité se colmateraient, les explosions du sens retourneraient à ses occlusions, où la femme naguère fragmentée et in-signfiante se lirait tout entière non plus entre les lignes mais outrageusement visible dans son intégralité et son intégrité. En somme, le bébé retournerait dans l'utérus par la magie de la marche arrière et la littérature serait de nouveau enceinte de la fiction et près d'accoucher $d^{\prime}$ 'un ensemble qui ne serait plus morcelé. Malheureusement, ce n'est pas si simple.

L'expression 'post-modernité' consacre le concept de 'modernité' qui fait fureur depuis presque un siècle. Les deuxentités sont liées non seulement par leur opposition mais par tout ce que la deuxième doit à la première. D'où de nombreuses contradictions sur leur nature respective qui est loin de faire le consensus général. Comment en parler justement? Et comment nommer ce qui viendra après la postmodernité?

Modernité et post-modernité - qui concernent bien d'autres domaines que la littérature-sont des catégories définies par des intellectuels mâles, que se sont appropriées les écrivaines et les intellectuelles. Je ne suis pas sûre que les femmes doivent s'y commettre dans les mêmes termes et sur les mêmes terrains, sans risquer de rester dans les marges et $\mathrm{d}^{\prime}$ ajouter à la confusion véhiculée par le masculin, toujours omnipotent dans le cours de la langue et des idées.

Car on sait bien que les hommes ne peuvent accepter leur crépuscule sans tenir la terre entière en otage sous la menace del'hiver nucléaire. Le patriarcat ne peut voir s'affaisser les Caryatides qui soutiennent son temple, sans vouloir que, littéralement, le ciel nous tombe sur la tête à tous et surtout à toutes.

* Titre d'un tableau de Magritte, 1928. 
La modernité, tout en élevant des socles divers au sujet, en a briséla statue avec fracas. De sorte qu'elle nous a donné à voir une exposition de socles vides comme des stèles funéraires tronquées où même le nom du mort a disparu. Fragments absolus du sujet, cendres dispersées. La formule brève qui a donné le fragment, selon un principe sain de contestation contre la notion même de genre littéraire, est elle-même devenue un genre.

La post-modernité qui a sonné le glas du genre-ou plutôt qui a fait le constat d'une mort évidente - cherche les ensembles $d^{\prime}$ où les innombrables éléments isolés se sont détachés qui étaient en conflit entre eux. On notera chez elle des restaurations et des révélations de l'ordre $d u$ retour $d u$ pendule ou de rencontre du premier type. Gageons qu'elle retrouvera l'organigramme du patriarcat et n'aura plus qu'à reconstituer le temple de celui-ci, que, entre autres pratiques, l'écriture au féminin avait ébranlé. C'est d'ailleurs ce que l'actualité, secondée par la fiction et l'art, nous donne à lire, à voir et à entendre dans notre réalité quotidienne, par les temps qui courent.

Louky Bersianik 


\section{Les femmes ont une autre version de l'histoire}

\section{Women Have Another Version of History}

This disenchantment (la post-modernité) is a turning back on self which doesn't really accommodate feminist thinking. This is an era of loose ends where even feminist thinking is viewed reductively. I think women have another version of history to write.

A partir du moment où un écrivain a pu déclarer qu'il était naturel d'être moderne, il m'est apparu que nous étions passés ailleurs, dans la post-modernité. La post-modernité invite à la reproduction des formes littéraires et au statu quo dans la pensée car il semble que nous ne puissions plus inventer l'avenir. Le désenchantement postmoderne s'accompagne d'une attitude de repli dont s'accommode mal la pensée féministe. Nous vivons une époque où les livres s'accumulent, nous écrivons le monde jusque dans ses détails infimes sans le rendre plus signifiant. C'est l'époque du désoeuvrement.

Depuis quelques années, des essais écrits généralement par des hommes récusent le féminisme qu'ils perçoivent dans la suite du mouvement de libération sexuelle ou du marxisme. Le féminisme est un mouvement incontournable dont on réduit singulièrement la portée et surtout dont on appauvrit l' ampleur de la réflexion. Le nihilisme voisine la post-modernité.

Je pense encore que les femmes ont à écrire une autre version de l'histoire, qu'il n'y a pas eu et qu'il n'y a pas d'époque idéale pour écrire mais que notre angle de vision, notre posture va se modifier.

France Théoret 


\section{Une conscience posthistorique}

\section{A Post-History Consciousness}

Women writers had already rerouted la modernité - inasmuch as la modernité sought to evacuate the subject - by introducing a feminine ' $I$ ' in their fiction, by refusing an intellectualism which sought a neutral body. La post-modernité once more juxtaposes reason/feeling, poetry/prose, sublime/ridiculous. It doesn'trepresentanother progressive stage of history, but as the end of history, which women don't consider catastrophic....

La post-modernité. Une notion relativement récente dans le monde francophone, introduite par le biais de la philosophie, ${ }^{1}$ alors que le 'post-modernism' était déjà une réalité bien établie dans le monde anglophone. C'est dire que ces deux concepts ne sont pas nécessairement synonymes. Dans le domaine littéraire, la postmodernité devient pour nous une notion critique qui sert à définir la textualité par rapport à la modernité.

Qu'est-ce à dire? On sait qu'au Québec avec la Barre du Jour et les Herbes Rouges, comme en France avec le revue Tel Quel, s'est élaborée, dans les années 1960 et 1970, une modernité qu'on pourrait taxer de formaliste, une modernité radicale s'efforçant de penser l'écriture en dehors d'une vision théologique du langage. On refusait de considérer plus longtemps l'auteur comme une sorte de démiurge, maître tout-puissant de son imaginaire. On voyait le texte comme produit de l'inconscient et de l'Histoire, travail du matériau linguistique d'où il fallait évacuer le sujet et le référent.

Mais très tôt, les écrivaines se sont montrées dissidentes par rapport à la modernité, en voulant réintroduire, dans leurs fictions, un jefemme. L'insertion de cette subjectivité a permis d'opérer un détournement de la modernité, de redonner à un formalisme qui risquait de tourner à vide, de dévenir fétichiste, un existentiel: la réalité des femmes avec leur quotidien, leur souffrance, la folie, la maternité, l'amour, le rêve, l'espoir.

Détournement, déplacement de la modernité. Par cette conscience féministe, la nouvelle écriture est sortie de son radicalisme orthodoxe pour se penser différemment, en dehors de la rupture absolue, en 
dehors d'un intellectualisme cherchant la neutralité du corps, l'occultation du je et du sens. Car la post-modernité est justement cet autre regard, cet autre point de vue sur l'écriture qui opère plutôt par addition que par soustraction ou discrimination. Elle place ensemble la raison et le sentiment, l'archaïque et le moderne, le beau et le laid, le sublime et le monstrueux, le poétique et le prosaïque, le fictif, l'anecdotique et le théorique. Elle est marquée d'une réappropriation des mythes (l'Amazone chez Nicole Brossard, le Grande Déesse Mère chez Jovette Marchessault, l'androgynie chez Yolande Villemaire, certaines figures chrétiennes chez Carole Massé, etc.) et des 'genres' littéraires donnés comme traditionnellement féminins: le journal, la lettre, la complainte, le monologue. Elle récupère des éléments de courants littéraires passés, rapatriant certains traits du classicisme, du baroque, de la conscience nationale, voire du folklore.

C'est donc dire que le post-modernité est, avant tout, liberté, audace, invention, sortie des cadres. Alors que la modernité croyait à la notion de progrès, de dépassement, le post-moderne 'se caractérise non seulement comme nouveauté par rapport au moderne, mais plus radicalement comme dissolution de la catégorie de nouveau, comme expérience d'une "fin de l'histoire", et non plus comme la présentation d'un autre stade, plus progressif ou plus régressif peu importe, de cette même histoire' ${ }^{2}$

Or c'est précisément à la fin de l'histoire que nous convie le féminisme, une pensée en dehors du patriarcat se situant dans une conscience posthistorique. Car plutôt qu'une idéologie - comme le marxisme par exemple -, le féminisme est une philosophie mouvante, ouverte, spiralique, qui met fin à la vision figée, fermée, unaire et linéaire qui prévaut depuis des millénaires et procède par exclusions. Sans fermer les yeux sur la menace nucléaire, le féminisme permet d'envisager la 'fin de l'histoire' en dehors de la catastrophe, d'associer post-modernité à possibilité. Par la recherche qui est la leur, on peut dire que, ici et maintenant, les écritures au féminin restent essentielles.

Louise Dupré

1 Je pense, par exemple, à la Condition postmoderne de Jean-François Lyotard, Paris, Minuit, coll. 'Critique', 1979, 109 pages, l'un des tout premiers texte où se voit traitée cette question en France.

2 Gianni Vatimo, la Fin de la modernité, Paris Seuil, coll. 'L'ordre philosophique', 1987, p. 10. 


\section{Pas au-delà? Ou faux pas?}

\section{A Step or a Stumble?}

I can't resist seeing la post-modernité with a certain relief: fictiontheory, which my English mother-tongue accommodates so badly, has been displaced in favour of new-narrative forms. Of course I see the danger in la post-modernité"s obsessivedesire to revive narrative ostensibly to mourn history, in fact the better to revive it. Still, the feminine ' $I$ ' born in la modernite of the last decade will blossom in the new fictional possibilities opening before her in this so-called end of an era. For a writer can make what she wants of an era, except ignore it.

Peut-être parce que je suis anglophone et que j'écris surtout en prose, le poids de la narration est toujours resté comme un surmoi, audessus de mon épaule. Je n'ai pas vécu de façon aussi intense que mes collègues québécois l'expérience du texte, cet amalgame de fiction et de théorie que signait surtout la modernité d'ici. Mais si, pour moi,les genres roman et nouvelle $n^{\prime}$ ont pas tout à fait éclaté, ils ont été profondément modifié sous l'influence de cette modernité. Et puis, dernièrement, la post-modernité est venue 'confirmer' la dissolution de ces formes en annonçant la mort du genre.Sauf que, évidemment, il fallait tout de suite remplir le vide avec autre chose. Et voici que surgissent de nouvelles formes baroques dans lesquelles la narrativité refait surface-pour raconter la fin del'Histoire, tout en s'accrochant à cette Histoire de façon obsessive. C'est une conjoncture qui me convient particulièrement bien (de point de vue stylistique)... et qui neme convient pas du tout (dans son rapport obsessif à l'Histoire).

Je pense que je partage cette ambivalence face à la post-modernité avec d'autres écrivaines. D'une part, ce sont les femmes qui ont brillé dans le récit, forme narrative qui a assimilé certaines leçons de la modernité tout en insistant sur l'émergence d'un je au féminin. Autrement dit, nous étions 'post-modernes' dans notre désir de créer de nouvelles forme narratives pour mettre en scène ce je au féminin bien avant que l'ère post-moderne soit 'née'. Certaines d'entre nous pourraient aussi apprécier le fait que le post-moderne joue très fortement sur le côté désespoir de la médaille. Même si nous, femmes, risquons de voir dans ce tragique des signes avant-courreurs d'une nouvelle 
époque. Je ne suis pas seule à penser qu'on vit, à cette fin du 20e siècle, la fin de la civilisation gréco-judéo-chrétienne.

Oui, peut-être sommes-nous en train de faire, nous femmes, ce qu'on avait fait par rapport à la modernité: c'est-à-dire créer un postmoderne au féminin. Un post-moderne qui signera cette époque à notre façon. Où les hommes voient la mort, on ne verra que la mort du patriarcat; ou les hommes voient clôture, on ne verra que la fin d'un cycle. Après tout, nous ne pouvons pas nous attendre à ce que le patriarcat soit mortellement blessé sans quelques cris au désastre.

C'est vrai que le côté réflexif de la modernité, tellement important pour l'émergence du je-féminin, semble se déplacer. Mais peut-être ce 'je' avait-elle besoin aussi de se faire aérer dans d'autres fictions. Peut-être a-t-elle besoin, par exemple, d'aller rejoindre d'autres femmes et hommes dans le carnaval en bas. Une chose est sûre: une écrivaine peut faire de chaque époque exactement ce qu'elle en veut.

Sauf l'ignorer.

Gail Scott 